\title{
QUALIDADE DE VIDA E PERCEPÇÃO ESTÉTICA DA CÁRIE DENTÁRIA
}

\section{Quality of life and aesthetic perception of dental caries \\ Calidad de vida y la percepción estética de la caries dental}

\section{Paula Reginne Vieira}

Universidade Estadual Paulista - FOAr/UNESP - Araraquara (SP) - Brasil

Camila Maria Bullio Fragelli

Universidade Estadual Paulista - FOAr/UNESP - Araraquara (SP) - Brasil

Fabiano Jeremias

Universidade Estadual Paulista - FOAr/UNESP - Araraquara (SP) - Brasil

\section{Lourdes Aparecida Martins dos Santos-Pinto}

Universidade Estadual Paulista - FOAr/UNESP - Araraquara (SP) - Brasil

\section{RESUMO}

Objetivo: Relacionar a percepção estética e a qualidade de vida relacionada à saúde bucal na presença de cárie dentária em escolares. Métodos: Estudo transversal realizado em Araraquara, São Paulo, Brasil, entre outubro de 2014 e março de 2015, com 260 escolares, de 8 a 10 anos, divididos em grupos clínicos segundo a presença de cárie dentária. Utilizaram-se questionários "Child Perceptions Questionnaire" e "Child Questionnaire About Teeth Appearence" e avaliação clínica para detecção de dentes cariados, perdidos ou obturados em dentes permanentes e decíduos. Dados analisados através do teste qui-quadrado e Mann Whitney, $\mathrm{p} \leq 0.05$. Resultados: Dos 260 escolares, 130 $(50 \%)$ apresentavam a doença cárie, com maior frequência $(62,0 \%)$ na faixa etária 8 anos. Variáveis socioeconômicas, como menor renda familiar e menor escolaridade da mãe, estiveram associadas ao grupo com cárie ( $\mathrm{p}<0,05$ e $\mathrm{p}<0,01$ respectivamente). No grupo com cárie, os domínios sintomas orais (4,9 vs 6,6), bem-estar emocional (3,0 vs 4,7) e bem-estar social $(2,2$ vs 3,5) foram maiores que no grupo sem cárie. Com relação à percepção estética, o domínio psicológico $(0,94$ vs 1,27$)$ e a percepção geral de saúde bucal $(2,26$ vs 2,93$)$ se apresentaram maiores no grupo com cárie. Na regressão linear, observou-se contribuição significativa dos números de dentes permanentes cariados e o índice ceo-d (dentes decíduos cariados, extraídos e obturados) na pior percepção de qualidade de vida. Conclusão: A cárie dentária afeta negativamente a percepção estética e a qualidade de vida em crianças de 8 a 10 anos.

Descritores: Qualidade de Vida; Estética Dentária; Criança; Cárie Dentária.

\begin{abstract}
Objetive: To relate the aesthetic perception and the oral health-related quality of life in the presence of dental caries in schoolchildren. Methods: Cross-sectional study carried out in Araraquara, São Paulo, Brazil, from October 2014 to March 2015, with 260 schoolchildren aged 8 to 10 years, divided into clinical groups according to the presence of dental caries. The Child Perceptions Questionnaire and the Child Questionnaire About Teeth Appearance were applied and a clinical evaluation of permanent and deciduous dentition was conducted for detection of decayed, missing or filled teeth. Data was analyzed using the chi-square test and Mann-Whitney's test, $p \leq 0.05$. Results: Of the 260 students, 130 (50\%) had caries disease, with the highest frequency (62.0\%) in 8-year-olds. Socioeconomic variables, such as lower family income and lower maternal schooling, were associated with the dental caries group ( $p<0.05$ and $p<0.01$, respectively). In the dental caries group, the domains oral symptoms (4.9 vs 6.6), emotional well-being (3.0 vs 4.7) and social well-being (2.2 vs 3.5$)$ were higher than in the caries-free group. Regarding the aesthetic perception, the psychological domain (0.94 vs 1.27) and the overall perception of oral health (2.26 vs 2.93) were higher in the group with dental caries. In the linear regression, there was a significant contribution of the number of permanent decayed teeth and the dmft (decayed, missing and filled deciduous teeth) index to the worst perception of quality of life. Conclusion: Dental caries negatively affects aesthetic perception and quality of life in 8- to 10-year-old children.
\end{abstract}

Descriptors: Quality of Life; Esthetics, Dental; Child; Dental Caries. 


\section{RESUMEN}

Objetivo: Relacionar la percepción estética y la calidad de vida relacionada con la salud bucal de escolares con caries dental. Métodos: Estudio transversal realizado en Araraquara, São Paulo, Brasil, entre octubre de 2014 y marzo de 2015 con 260 escolares entre 8 y 10 años que fueron divididos en grupos clínicos según la presencia de caries dental. Se utilizaron los cuestionarios "Child Perceptions Questionnaire" y "Child Questionnaire About Teeth Appearence" y la evaluación clinica para detectar los dientes con caries, los perdidos $u$ con obturaciones en dientes permanentes y deciduos. Los datos fueron analizados a través de las pruebas Chi-cuadrado y Mann Whitney con $p \leq 0$,05. Resultados: Entre los 260 escolares, 130 (50\%) tenían caries con más frecuencia en la franja de edad de 8 años (62,0\%). Las variables socioeconómicas como la menor renta familiar y menor escolaridad de la madre se asociaron con el grupo que tenía caries ( $p<0,05$ y $p<0,01$ respectivamente). Los dominios síntomas orales $(4,9$ vs 6,6), el bien estar emocional $(3,0$ vs 4,7) y el bien estar social (2,2 vs 3,5) fueron mayores en el grupo con caries que el grupo sin caries. Respecto la percepción estética, el dominio psicológico $(0,94$ vs 1,27) y la percepción general de salud bucal $(2,26$ vs 2,93) se presentaron mayores en el grupo con caries. A partir de la regresión linear se observó la contribución significativa de los números de dientes permanentes con caries y el índice ceo-d (dientes deciduos con caries, extraídos y con obturación) para la peor percepción de calidad de vida. Conclusión: La caries dental afecta de manera negativa la percepción de la estética y la calidad de vida de niños entre 8 y 10 años.

Descriptores: Calidad de Vida; Estética Dental; Niño; Caries Dental.

\section{INTRODUÇÃO}

Os conceitos contemporâneos de saúde bucal sugerem que ela deve ser uma parte integrante da saúde geral e do bem-estar. A saúde bucal é multifacetada e inclui, sem limitações, a capacidade de falar, sorrir, cheirar, saborear, tocar, mastigar, engolir e transmitir uma variedade de emoções, através de expressões faciais, com confiança e sem dor, ou desconforto, e sem doença do complexo craniofacial. A condição bucal reflete os atributos fisiológicos, sociais e psicológicos que formam a essência da qualidade de vida e é influenciada pelas experiências, percepções, expectativas e capacidade de se adaptar a circunstâncias em constantes transformações ${ }^{(1)}$

A cárie dentária é a doença bucal mais prevalente e, embora em declínio, ainda atinge $56 \%{ }^{(2)}$ da população brasileira aos 12 anos. Por ser uma doença multifatorial, deve ser compreendida considerando seu impacto funcional e psicossocial.

A promoção de saúde bucal durante a infância é essencial, tendo em vista que os impactos da doença cárie não estão apenas relacionados às experiências atuais, mas também às experiências de saúde bucal do passado e às implicações ao longo da vida ${ }^{(3)}$. $\mathrm{O}$ autocuidado desde os anos da infância implica em uma melhor saúde bucal na vida adulta e menor impacto bio-psicossocial ${ }^{(4)}$ beliefs about oral health care (held by individuals and their parents Somadas ao exame clínico, a avaliação da Qualidade de Vida Relacionada à Saúde Bucal (QVRSB) $)^{(5)}$ e a avaliação da percepção estética ${ }^{(6)}$ mensuram os impactos funcionais e psicossociais das doenças bucais, auxiliando os profissionais na compreensão integral do paciente.

Na avaliação da QVRSB, aspectos como o impacto funcional, os sintomas orais, o bem-estar social e o bem-estar emocional são avaliados ${ }^{(5)}$, e a literatura aponta uma forte associação da presença da cárie dentária com impactos na qualidade de vida em crianças ${ }^{(6,7)}$, principalmente na presença de lesões de cárie não tratadas ${ }^{(4)}$ beliefs about oral health care (held by individuals and their parents e em situação de vulnerabilidade social ${ }^{7,8)}$.

A avaliação da percepção estética considera aspectos individuais autorreportados relacionados à aparência, coloração dentária, ao posicionamento dentário e à saúde bucal e seu impacto na satisfação com a aparência ${ }^{(6)}$. Embora alguns pontos sejam apresentados na QVRSB, a dimensão desse impacto não é claramente abordada, de forma que pesquisas nessa temática devem ser encorajadas com o intuito de entender toda a extensão da doença.

Sendo assim, para melhor entender a complexidade da doença cárie, o objetivo desta pesquisa foi relacionar a percepção estética e a qualidade de vida da saúde bucal à presença de cárie dentária em escolares.

\section{MÉTODOS}

O desenvolvimento deste estudo transversal ocorreu no período de outubro de 2014 à março de 2015, na cidade de Araraquara, São Paulo, Brasil.

Os dados foram coletados em escolas da rede pública e privada, sendo convidadas a participar do estudo crianças, com idades entre 8 e 10 anos, matriculados naquele ano no ensino fundamental, estratificados em escolares da rede pública (217) e privada (43). Utilizou-se amostragem por conveniência.

Após sorteio, selecionaram-se sete escolas, totalizando 406 escolares cujos responsáveis autorizaram a participação no estudo. Como critérios de inclusão, os escolares deveriam estar matriculados no ensino fundamental, alfabetizados, terem entre 8 e 10 anos, terem autorização de seus responsáveis legais e concordar em participar como voluntários. Tomaram-se como 
critérios de exclusão: presença de dentes anteriores fraturados devido a trauma e escolares afetados por defeitos de esmalte e uso de aparelhos ortodônticos. Assim, o total de 260 escolares foram incluídos nas análises.

Realizou-se o presente estudo em três etapas: (i) contato com os responsáveis pelos escolares; (ii) aplicação dos questionário de QVRSB e percepção estética; (iii) exame clínico dentário.

A primeira etapa foi realizada utilizando informações fornecidas pelas escolas incluídas no estudo, na qual se enviou aos responsáveis a autorização para participar da pesquisa e o questionário para coletar as variáveis socioeconômicas através de perguntas semi-estruturadas baseadas na Pesquisa Nacional de Amostra por Domicilio (PNAD) ${ }^{(9)}$.

Na segunda etapa, após a autorização dos responsáveis, aplicaram-se os questionários "Child Perceptions Questionnaire" (CPQ), na versão longa de 29 questões para crianças de 8 a 10 anos $^{(8)}$, e o Child Perceptions Questionnaire About Teeth Appearance (CQATA), com 5 questões divididas em 12 subitens (8), validados na língua portuguesa ${ }^{(9)}$.

O CPQ é um conjunto de instrumentos de medida, validado na língua portuguesa ${ }^{(10)}$, que leva em consideração as habilidades cognitivas e o dia a dia. Esse instrumento abrange quatro domínios: sintomas orais, limitações funcionais, bem-estar emocional e bem-estar social.

O CQATA possui versão traduzida e validada para o português brasileiro ${ }^{(6)}$, e compreende questões de ordem física, psicológica e social, além das percepções sobre as alterações de cor e outras condições de ordem estética, com respostas de múltipla escolha para seus itens e subitens.

Aplicou-se os questionários CPQ e CQATA em sala de aula, em versão impressa previamente ao exame clínico sob a supervisão dos pesquisadores. As crianças responderam às questões individualmente. Quanto maior o escore somado nas respostas de múltipla escolha, maior o impacto da saúde bucal na qualidade de vida e percepção estética do infante ${ }^{(11)}$.

$\mathrm{Na}$ terceira etapa, um único examinador, previamente treinado para detecção de dentes cariado, perdido ou obturado em dentes permanentes e cariados e obturados em dentes decíduos, segundo os critérios da Organização Mundial de Saúde ${ }^{(12)}$, por meio dos índices CPOD (kappa $=0,88)$ e ceo-d (kappa $=0,92)$, respectivamente, avaliou as crianças clinicamente.

Para a realização das análises estatísticas, dividiu-se a amostra de acordo com os grupos clínicos: grupo controle (ausência de cárie dentária) e grupo caso (presença de cárie dentária), divididos através dos dados obtidos segundo os indices CPO-D e ceo-d. Utilizou-se os pacotes estatísticos SPSS, versão 22.0, para a análise estatística do estudo, sendo o nível de significância considerado de $\alpha=0,05$. Os escores do CPQ e CQATA apresentaram-se de forma anormal depois de verificada por meio do teste de Kolmogorov-Smirnov. Empregaram-se os testes qui-quadrado/partição para verificar a distribuição da amostra de acordo com as variáveis independentes (sexo, idade, escola) para cada grupo clínico. Dicotomizou-se as variáveis socioeconômicas e relacionaram-se as características clínicas através do teste qui-quadrado.

Considerando as opções de resposta disponíveis, realizou-se a soma dos escores do CPQ e CQATA para cada domínio do instrumento. Os dados omissos ou perdidos foram imputados pela média obtida no domínio para cada indivíduo ${ }^{(13)}$. Utilizou-se o teste de Mann Whitney para verificar a diferença nos domínios do CPQ e do CQATA, de acordo com as características clínicas.

Mensurou-se pela regressão linear a associação entre as médias de todos os domínios de ambos os questionários e outros 7 fatores. Os seguintes fatores foram selecionados: número de dentes decíduos cariados e obturados; número de dentes permanentes cariados, perdidos e obturados; e ainda os índices de CPO-D e ceo-d. As variáveis eficazes entraram no modelo quando $\mathrm{p} \leq 0.05$, através do método stepwise.

Respeitaram-se os aspectos éticos durante o desenvolvimento da presente pesquisa, a qual foi submetida à avaliação do Comitê de Ética em Pesquisa da Faculdade de Odontologia de Araraquara, Universidade Estadual Paulista (CEP-FOARUNESP), e foi executada somente após a aprovação, conforme a Resolução n ${ }^{\circ}$ 466/12 do Conselho Nacional de Saúde (Parecer $\left.\mathrm{n}^{\mathrm{o}} 070020 / 2013\right)$.

\section{RESULTADOS}

Metade da amostra avaliada apresentou a doença cárie $(50,0 \% / \mathrm{n}=130)$, sendo a maior frequência encontrada em crianças de 8 anos (62,0\%), como demonstra a Tabela I. Dentre os escolares com doença cárie, encontrou-se um índice ceo-d de 1,03 e CPO-D 0,37. Na Figura 1, os índices ceo-d e CPO-D estão divididos em dentes cariados, perdidos e obturados, segundo a idade.

Na Tabela II, a presença de doença cárie no grupo caso, esteve associada às variáveis socioeconômicas: renda familiar inferior a três salários mínimos e escolaridade materna inferior ao primeiro grau completo. 
Tabela I - Distribuição da amostra de acordo com o sexo, idade, escola e subgrupos clínicos, controle $(\mathrm{CPO}-\mathrm{D} / \mathrm{ceo}-\mathrm{d}=0)$ e caso (CPO-D/ceo-d $\neq 0$ ). Araraquara, São Paulo, 2014-2015.

\begin{tabular}{lcc}
\hline & Controle & Grupos Clínicos \\
\hline n (\%) & $130(50,0)$ & Caso \\
Sexo & & $130(50,0)$ \\
$\quad$ Masculino & $61(51,7)$ & \\
Feminino & $69(48,6)$ & $57(48,3)$ \\
Idade & & $73(51,4)$ \\
8 & $35(38,0)$ & $57(62,0)^{\mathrm{a}}$ \\
9 & $49(51,0)$ & $47(49,0)^{\mathrm{ab}}$ \\
10 & $46(63,9)$ & $26(36,1)^{\mathrm{b}}$ \\
Escola & & $115(53,0)$ \\
Pública & $102(47,0)$ & $15(34,9)$ \\
Particular & $28(65,1)$ & ${ }^{\mathrm{a}} \mathrm{p}<0,05$ \\
\hline
\end{tabular}

CPO-D/ceo-d: número de dentes cariados, perdidos e obturados, decíduos e permanentes. ${ }^{\text {a }}$ teste Qui-quadrado; ${ }^{\text {b }}$ teste Qui-quadrado partição.

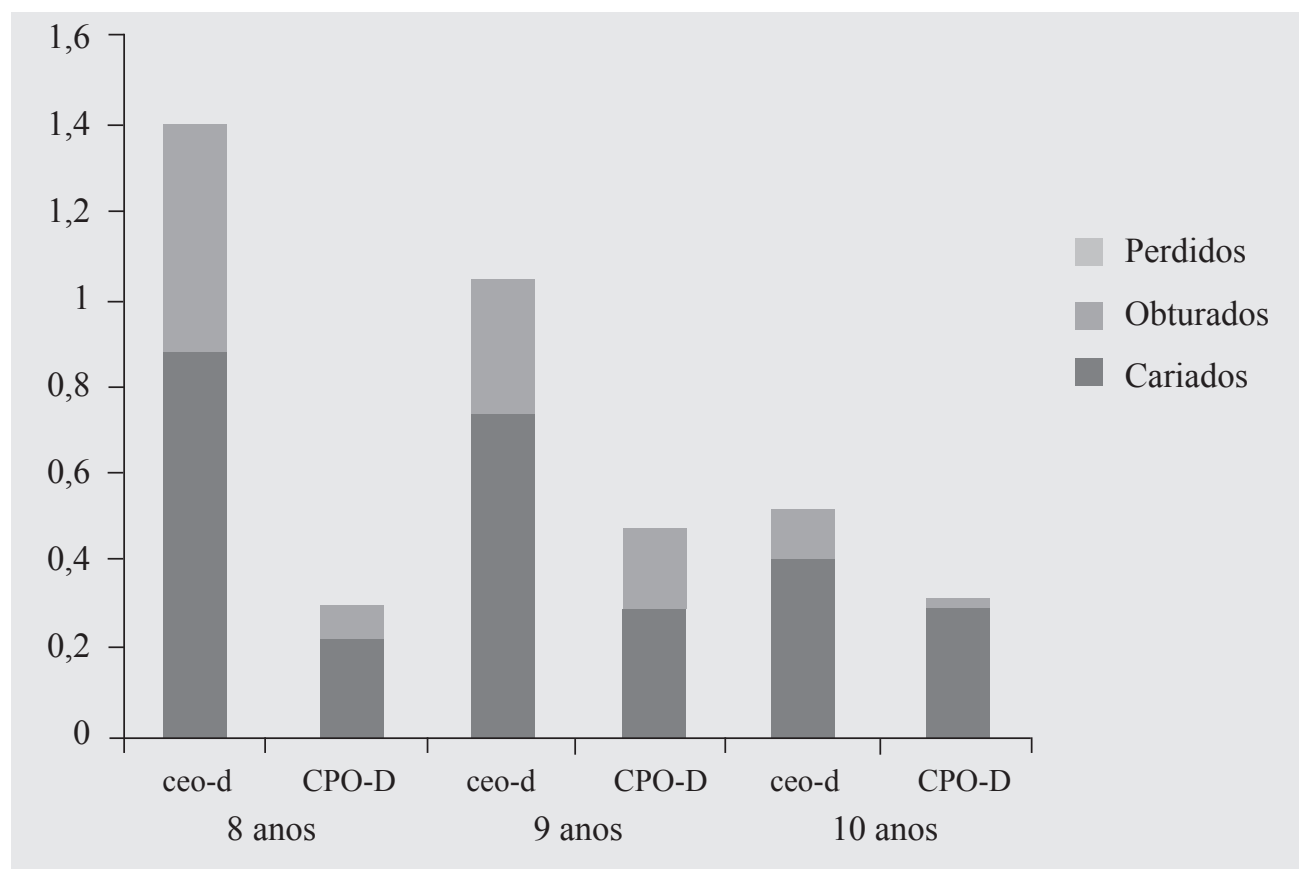

Figura 1 - CPO-D e ceo-d segundo o número de dentes perdidos, obturados e/ou cariados, segundo a idade. Araraquara, São Paulo, 2014-2015.

Legenda: CPO-D/ceo-d: número de dentes cariados, perdidos e obturados, permanentes e decíduos, respectivamente. 
Tabela II - Variáveis socioeconômicas relacionadas aos grupos clínicos, controle $(\mathrm{CPO}-\mathrm{D} / \mathrm{ceo}-\mathrm{d}=0)$ e caso $(\mathrm{CPO}-\mathrm{D} / \mathrm{ceo}-\mathrm{d} \neq$ 0). Araraquara, São Paulo, 2014-2015.

\begin{tabular}{lccc}
\hline Variáveis & Controle & Caso & p \\
\hline Renda Familiar & & & \\
$\quad$ Inferior à 3 salários mínimos & $82(45,1)$ & $100(54,9)$ & $\mathrm{p}<0,05^{\mathrm{a}}$ \\
$\quad$ Superior à 3 salários mínimos & $42(60,9)$ & $27(39,1)$ & \\
Escolaridade do pai & & & \\
$\quad$ Analfabeto até $1^{\circ}$ grau completo & $80(47,6)$ & $88(52,4)$ & \\
$2^{\circ}$ grau incompleto até ensino superior & $35(61,4)$ & $22(38,6)$ & \\
Escolaridade da mãe & & & \\
$\quad$ Analfabeta até $1^{\circ}$ grau completo & $10(27,8)$ & $26(72,2)$ & $\mathrm{p}<0,01^{\mathrm{a}}$ \\
$2^{\circ}$ grau incompleto até ensino superior & $115(53,2)$ & $101(46,8)$ & \\
\hline
\end{tabular}

CPO-D/ceo-d: número de dentes cariados, perdidos e obturados, decíduos e permanentes. ${ }^{a}$ teste Qui-quadrado

Na Tabela III é possível observar as diferenças entre os grupos clínicos caso e controle e os domínios do questionário CPQ 8-10. No escore total ( 12,3 vs 17,6$)$, sintomas orais ( 4,9 vs 6,6$)$, bem-estar emocional ( 3,0 vs 4,7$)$ e bem-estar social $(2,2$ vs 3,5 ), o grupo clínico caso, apresentou valores maiores do que o controle $(\mathrm{p}<0,05)$.

Ainda na Tabela III, ao comparar os grupos clínicos, caso e controle, com o questionário CQATA, observou-se que, no grupo clínico caso, a percepção geral de saúde bucal $(2,26$ vs 2,93$)$, domínio psicológico $(0,94$ vs 1,27$)$, percepção da aparência $(1,76$ vs 2,14$)$ e a percepção da saúde dentária $(1,36$ vs 1,89$)$ são maiores que no grupo controle $(\mathrm{p}<0,05)$.

Os resultados da regressão linear (Tabela IV) mostram associação entre o ceo-d e o número de dentes permanentes cariados com os domínios dos questionários, sendo que, para cada dente permanente a mais cariado, há um aumento médio de 1,0 no bemestar emocional e social do CPQ, e 0,5 na percepção geral de saúde bucal do CQATA.

Tabela III - Média ( $( \pm \mathrm{DP})$ dos domínios dos questionários CPQ e CQATA de acordo com os grupos clínicos, controle (CPO-D/ ceo-d $=0)$ e caso $($ CPO-D/ceo-d $\neq 0$ ). Araraquara, São Paulo, 2014-2015.

\begin{tabular}{lccc}
\hline & \multicolumn{2}{c}{ Grupos Clínicos } & \\
\cline { 2 - 3 } Domínios & $\begin{array}{c}\text { Controle } \\
\mathbf{n}=\mathbf{1 3 0}\end{array}$ & $\begin{array}{c}\text { Caso } \\
\mathbf{n}=\mathbf{1 3 0}\end{array}$ & $\mathbf{p}$ \\
\hline Domínios do CPQ & & & \\
Escore total do CPQ8-10 [0-100] & & & \\
Sintomas orais [0-20] & $12,3(10,5)$ & $17,6(14,9)$ & $\mathrm{p}<0,001$ \\
Limitaçães funcionais [0-20] & $4,9(3,2)$ & $6,6(4,1)$ & $\mathrm{p}<0,001$ \\
Bem-estar emocional [0-20] & $2,2(2,7)$ & $2,9(3,4)$ & \\
Bem-estar social [0-40] & $3,0(3,5)$ & $4,7(5,1)$ & $\mathrm{p}<0,01$ \\
Domínios do CQATA & $2,2(3,6)$ & $3,5(5,0)$ & $\mathrm{p}<0,05$ \\
Percepção geral da saúde bucal [0-9] & & & \\
Domínio físico [0-3] & $2,26(2,26)$ & $2,93(2,46)$ & $\mathrm{p}<0,05$ \\
Domínio psicológico [0-3] & $0,82(1,00)$ & $1,03(1,12)$ & \\
Domínio Social [0-3] & $0,94(0,99)$ & $1,27(1,14)$ & $\mathrm{p}<0,05$ \\
Percepção da aparência [0-5] & $0,50(0,97)$ & $0,62(1,01)$ & \\
Percepção da posição [0-5] & $1,76(1,30)$ & $2,14(1,40)$ & $\mathrm{p}<0,05$ \\
Percepção da coloração [0-5] & $2,70(1,44)$ & $2,59(1,52)$ & \\
Percepção da saúde dentária [0-5] & $2,13(1,22)$ & $2,27(1,31)$ & \\
Satisfação com aparência [0-4] & $1,36(1,22)$ & $1,89(1,44)$ & $\mathrm{p}<0,05$ \\
\hline
\end{tabular}

CPO-D/ceo-d: número de dentes cariados, perdidos e obturados, decíduos e permanentes; CPQ: child perceptions questionaire; CQATA: child perceptions questionaire about teeth appearence. [ ]: possível variação dos escores. Quanto maior o escore, mais negativa a qualidade de vida relacionada à saúde bucal e a percepção estética. Mann Whitney Test. 
Tabela IV - Análise de regressão pelo método stepwise: variáveis independentes como preditoras do CPQ (Child perceptions questionnaire) e do CQATA (child perceptions questionaire about teeth appearence). Araraquara, São Paulo, 2014-2015.

\begin{tabular}{|c|c|c|c|}
\hline & $\begin{array}{c}\text { Análise de regressão } \\
\text { B }[95 \% \mathrm{CI}]\end{array}$ & p-value & $\mathbf{R}^{2}$ \\
\hline \multicolumn{4}{|l|}{ CPQ } \\
\hline \multicolumn{4}{|l|}{ Escore total } \\
\hline ceo-d & $1,405[0,457-2,353]$ & 0,004 & 0,056 \\
\hline Permanentes cariados & $2,263[0,040-4,486]$ & 0,046 & 0,056 \\
\hline \multicolumn{4}{|l|}{ Sintomas orais } \\
\hline ceo-d & $0,395[0,122-0,668]$ & 0,005 & 0,031 \\
\hline \multicolumn{3}{|l|}{ Limitações funcionais } & 0,026 \\
\hline \multicolumn{4}{|l|}{ Bem-estar emocional } \\
\hline ceo-d & $0,465[0,148-0,781]$ & 0,004 & 0,068 \\
\hline Permanentes cariados & $1,002[0,260-1,745]$ & 0,008 & 0,068 \\
\hline \multicolumn{4}{|l|}{ Bem-estar social } \\
\hline Permanentes cariados & $1,027[0,283-1,770]$ & 0,007 & 0,044 \\
\hline Decíduos restaurados & $0,668[0,034-1,303]$ & 0,039 & 0,044 \\
\hline \multicolumn{4}{|l|}{ CQATA } \\
\hline \multicolumn{4}{|l|}{ Percepção geral da saúde bucal } \\
\hline Permanentes cariados & $0,577[0,169-0,984]$ & 0,006 & 0,029 \\
\hline \multicolumn{4}{|l|}{ Domínio físico } \\
\hline Permanentes cariados & $0,213[0,030-0,397]$ & 0,023 & 0,020 \\
\hline \multicolumn{4}{|l|}{ Domínio psicológico } \\
\hline Permanentes cariados & $0,329[0,145-0,512]$ & 0,001 & 0,046 \\
\hline ceo-d & $0,134[0,035-0,233]$ & 0,008 & 0,027 \\
\hline \multicolumn{4}{|l|}{ Percepção da coloração } \\
\hline Decíduos restaurados & $0,205[0,020-0,389]$ & 0,030 & 0,018 \\
\hline $\begin{array}{l}\text { Percepção da saúde dentária } \\
\text { ceo-d }\end{array}$ & $0,166[0,068-0,263]$ & 0,001 & 0,042 \\
\hline
\end{tabular}

CPQ: Child Perceptions Questionnaire; CQATA: child perceptions questionaire about teeth appearence; ceo-d: número de dentes decíduos cariados, perdidos e obturados.

\section{DISCUSSÃO}

A cárie é uma doença multifatorial, que não se limita apenas a alteração da estrutura dental ${ }^{(14)}$ caries (International Caries Detection and Assessment System, ICDAS II). Essa doença causa sintomatologia dolorosa e alterações na linguagem oral ${ }^{(15)}$ missing, and filled teeth--D-DMFT in permanent teeth or d-dfmt in primary teeth, assim como impacta a qualidade de vida e a percepção estética, devido aos sinais e sintomas apresentados, como observado no presente estudo.

Associar as medidas de QVRSB e percepção estética com indicadores clínicos de saúde bucal permite avaliar as reais necessidades de saúde bucal do individuo. A literatura aponta que, quando utilizados em conjunto, podem beneficiar o planejamento de serviços odontológicos, uma vez que os achados clínicos sozinhos geralmente superestimam as necessidades do paciente ${ }^{(14)}$. Além disso, os indicadores sócio-odontológicos podem ser usados para priorizar o tratamento odontológico em situações de falta de recursos. De acordo com esse raciocínio, se não houver nenhum impacto na qualidade de vida ou percepção estética, não há necessidade de intervenção clínica imediata, e o paciente pode ser dirigido para um programa de educação saúde bucal ${ }^{(14)}$.

Assim, o presente estudo avaliou a relação da presença de histórico prévio ou atual de cárie com o tipo de escola frequentada, a renda familiar e a escolaridade dos pais, encontrando associações significativas com a renda familiar inferior a 3 salários mínimos $(p<0,05)$ e escolaridade materna inferior ao primeiro grau $(p<0,01)$. Esse vínculo entre saúde e os resultados socioeconômicos pode ser explicado pelo fato da renda familiar ter um efeito direto sobre a capacidade de acesso a bens, serviços e outros recursos que promovem a saúde além dos impactos nos recursos psicossociais e psicológicos ${ }^{(4)}$.

As habilidades de enfrentamento sociais e psicológicas das crianças estão em fase de desenvolvimento, assim é necessário ampliar as investigações sobre o impacto da saúde bucal na qualidade de vida das crianças ${ }^{(16)}$. Sendo assim, o presente estudo abrangeu a faixa etária de 8 a 10 anos, apresentando prevalência de cárie significativa (50\%), com impacto significativo na QVRSB nos domínios sintomas orais e bemestar emocional e social, sendo que as maiores associações foram encontradas na presença de permanentes cariados e maiores índices de ceo-d segundo a regressão linear. 
A associação de piores índices de QVRSB e a cárie dentária também foi relatada no Sul do Brasil, em pesquisa realizada com 1528 crianças brasileiras de 12 anos, sendo que a presença de uma única cavidade cariada já foi associada a uma pior QVRSB $^{(17)}$. Nesse mesmo estudo, observou-se, nas crianças com dentes cariados cavitados, um impacto oral sobre as atividades diárias devido aos sintomas orais apresentados, tais como dor de dente, mau hálito e impactação alimentar ${ }^{(17)}$.

Com a proximidade da adolescência, a preocupação das crianças sobre a sua aparência estética torna-se mais significativa, como aponta o estudo realizado no Rio de Janeiro, com 571 escolares. Os adolescentes que possuíam os dentes anteriores cariados tinham o domínio de percepção estética, segundo o questionário Child-Oral Impacts on Daily Performance (COIDP), mais afetado ${ }^{(18)}$. As crianças que sofreram prejuízo funcional na alimentação ou algum envolvimento emocional por causa da cárie dentária tiveram uma melhoria em sua qualidade de vida depois de receber tratamento odontológico ${ }^{(17)}$.

Resultados semelhantes aos encontrados nos estudos do sul do Brasil ${ }^{(17)}$ e do Rio de Janeiro ${ }^{(18)}$ foram encontrados no presente estudo. Pela regressão linear, observou-se que, para cada dente permanente a mais cariado, há um aumento médio de 1,0 nos domínios bem-estar emocional e social com relação à qualidade de vida, e de 0,5 na percepção geral de saúde bucal com relação à percepção estética.

Ainda no Brasil, um estudo realizado com 167 alunos, de 08 a 14 anos, de escolas públicas de Piracicaba-SP, observou que a experiência de doenças e distúrbios orais, bem como fenômenos psicológicos, como ansiedade e depressão, influenciaram a QVRSB ${ }^{(18)}$; assim como a presença de cárie dentária não tratada e suas consequências medidas através do PUFA/pufa index apresentou impactos sobre em todos os domínios do CPQ 8-10 (19).

No presente estudo, a percepção geral da saúde bucal, principalmente no domínio psicológico, na percepção da aparência e na percepção da saúde dentária, foi pior no grupo com cárie dentária (caso). Assim como observado no estudo realizado com 861 crianças de 4 anos, em que crianças com cárie severa afirmam com mais frequência que sentem dor ou ficam tristes com os seus dentes, quando comparadas com as crianças livres de cárie ${ }^{(20)}$. Além disso, os pais ou responsáveis das crianças com cárie relataram que seus filhos tinham vergonha de sorrir por causa de seus dentes $(31,2 \%)$, enquanto alguns $(9,1 \%)$ pararam de brincar com outras criançaspela mesma razão ${ }^{(20)}$.

Cirurgiões-dentistas reconhecem que o prejuízo estético afeta negativamente o desenvolvimento social e psicológico da criança $^{(21)}$. Todavia, tratamentos odontológicos no Brasil, embora em ascensão, não estão disponíveis a toda população. Cerca de 29,6 milhões de pessoas (18,7\% da população brasileira) nunca consultou um cirurgião-dentista, apresentando-se as maiores proporções nas crianças menores de 4 anos $(85,6 \%)^{(22)}$.

A necessidade de se promover a saúde bucal desde a primeira infância é imprescindível, uma vez que programas de saúde bucal na atenção primária podem produzir mudanças impactantes. Como a encontrada em crianças expostas a programas de saúde bucal frequentes nos primeiros anos de vida, onde houve uma menor frequência de cárie e uma melhor qualidade de $\operatorname{vida}^{(21)}$.

O estudo longitudinal, realizado na Holanda, com 6990 crianças acompanhadas desde o nascimento, observou que as crianças com cáries severas aos 6 anos tiveram maiores probabilidades de ter uma QVRSB mais baixa aos 10 anos de idade. O estudo destacou a importância da saúde bucal durante a infância, porque aqueles com um comprometimento precoce da saúde bucal são muito mais propensos a seguir uma trajetória que levará a uma má saúde bucal ${ }^{(3)}$.

Todavia, o presente estudo apresenta uma amostragem realizada por conveniência e sem base populacional. Embora indiquem uma direção, seus resultados devem ser lidos com cautela. A utilização dos índices CPO-D e ceo-d também limitam a interpretação dos dados, uma vez que a extensão e severidade das lesões de cárie não foram avaliadas. Sendo assim, novas pesquisas devem ser conduzidas a fim de nortear políticas públicas direcionadas para a saúde bucal infantil.

\section{CONCLUSÃO}

Conclui-se que a cárie dentária afeta tanto a qualidade de vida relacionada à saúde bucal quanto a percepção estética, em crianças de 8 a 10 anos.

\section{AGRADECIMENTOS}

Ao CNPq e à Faculdade de Odontologia de Araraquara.

\section{CONFLITOS DE INTRESSE}

Os autores declaram que não houve conflito de interesse na execução desta pesquisa.

\section{REFERÊNCIAS}

1. FDI World Dental Federation. FDI's definition of oral health [Internet]. [acesso em 2017 Dez 19]. Disponível em: http:// www.fdiworlddental.org/oral-health/fdis-definition-of-oral-health 
2. Ministério da Saúde (BR). SB Brasil 2010: Pesquisa Nacional de Saúde Bucal: resultados principais. Brasília: Ministério da Saúde; 2014 [acesso em 2017 Dez 19]. Disponivel em :http://pesquisa.bvsalud.org/bvsms/resource/pt/mis-36702

3. Kragt L, Van der Tas JT, Moll HA, Elfrink ME, Jaddoe VW, Wolvius EB, et al. Early caries predicts low oral health-related quality of life at a later age. Caries Res. 2016;50(5):471-9.

4. Broadbent JM, Zeng J, Foster Page LA, Baker SR, Ramrakha S, Thomson WM. Oral health-related beliefs, behaviors, and outcomes through the life course. J Dent Res [Internet]2016 [acesso em 2017 Dez 18];95(7):808-13. Disponível em: https://www.ncbi.nlm.nih.gov/pubmed/26936215

5. Martins MT, Sardenberg F, Vale MP, Paiva SM, Pordeus IA. Dental caries and social factors: impact on quality of life in Brazilian children. Braz Oral Res [Internet] 2015; [acesso em 2018 Dez 18]; 29(1):S1806-83242015000100310. Disponível em: https://www.ncbi.nlm.nih.gov/pubmed/26892354

6. Schuch HS, Costa FS, Torriani DD, Demarco FF, Goettems ML. Oral health-related quality of life of schoolchildren: impact of clinical and psychosocial variables. Int J Paediatr Dent [Internet]. 2015 [acesso em 2017 Dez 20];25(5):358-65. Disponível em: https://www.ncbi.nlm.nih.gov/pubmed/25047069

7. Instituto Brasileiro de Geografia e Estatística. Pesquisa Nacional por Amostra de Domicílios [acesso em 2017 Dez 20]. Disponível em: https://ww2.ibge.gov.br/home/estatistica/pesquisas/pesquisa_resultados.php?id_pesquisa=40

8. Jokovic A, Locker D, Tompson B, Guyatt G. Questionnaire for measuring oral health-related quality of life in eight- to tenyear-old children. Pediatr Dent [Internet]. 2004 [acesso em 2017 Dez 20]; 26(6):512-8 Disponível em: http://www.ncbi. nlm.nih.gov/pubmed/15646914.

9. Furtado GES, Sousa MLR, Barbosa TS, Wada RS, Martínez-Mier EA, Almeida MEL. Percepção da fluorose dentária e avaliação da concordância entre pais e filhos: validação de um instrumento. Cad Saúde Pública [Internet]. 2012 [acesso em 2017 Dez 18];28(8):1493-505. Disponível em: http://www.scielosp.org/scielo.php?script=sci_arttext\&pid=S0102-311X2 012000800008\&lng=pt\&nrm=iso\&tlng=pt

10. Barbosa TS, Vicentin MDS, Gavião MBD. Qualidade de vida e saúde bucal em crianças - Parte I: versão brasileira do Child Perceptions Questionnaire 8-10. Ciênc Saúde Colet [Internet]. 2011 [acesso em 2017 Dez 18];16(10):4077-85. Disponível em: http://www.scielosp.org/scielo.php?script=sci_arttext\&pid=S1413-81232011001100013\&lng=pt\&nrm=iso\&tlng=pt

11. Barbosa TS, Tureli MCM, Gavião MBD. Validity and reliability of the Child Perceptions Questionnaires applied in Brazilian children. BMC Oral Health [Internet]. 2009. [acesso em 2017 Dez 18]; 9:13. Disponível em: http://www.ncbi. nlm.nih.gov/pubmed/19450254

12. World Health Organization. Oral Health Surveys: Basic Methods. $5^{\text {th }}$ ed. Geneva: WHO; 2013.

13. Shrive FM, Stuart H, Quan H, Ghali WA. Dealing with missing data in a multi-question depression scale: a comparison of imputation methods. BMC Med Res Methodol [Internet]. 2006 [acesso em 2017 Dez 18];6:57. Disponível em: http:// www.ncbi.nlm.nih.gov/pubmed/17166270

14. García-Pérez Á, Irigoyen-Camacho ME, Borges-Yáñez SA, Zepeda-Zepeda MA, Bolona-Gallardo I, Maupomé G. Impact of caries and dental fluorosis on oral health-related quality of life: a cross-sectional study in schoolchildren receiving water naturally fluoridated at above-optimal levels. Clin Oral Investing [Internet]. 2017 [acesso em 2017 Dez 20];21(9):2771-80. Disponível em: https://www.ncbi.nlm.nih.gov/pubmed/28251432

15. Mota-Veloso I, Soares ME, Alencar BM, Marques LS, Ramos-Jorge ML Ramos-Jorge J. Impact of untreated dental caries and its clinical consequences on the oral health-related quality of life of schoolchildren aged 8-10 years. Qual Life Res [Internet]. 2016 [acesso em 2017 Dez 20];25(1):193-9. Disponível em: https://www.ncbi.nlm.nih.gov/pubmed/26135023

16. Pentapati KC, Acharya S, Bhat M, Krishna Rao SV, Singh S. Oral health impact, dental caries, and oral health behaviors among the National Cadets Corps in South India. J Investig Clin Dent [Internet]. 2013 [acesso em 2017 Dez 20]; 4(1):3943. Disponível em: http://doi.wiley.com/10.1111/j.2041-1626.2012.00134.x

17. Alves LS, Damé-Teixeira N, Susin C, Maltz M. Association among quality of life, dental caries treatment and intraoral distribution in 12-year-old South Brazilian schoolchildren. Community Dent Oral Epidemiol [Internet]. 2013 [acesso em 2017 Dez 20];41(1):22-9. Disponível em: http://doi.wiley.com/10.1111/j.1600-0528.2012.00707.x

18. Souza Barbosa T. Health-related quality of life in children and preadolescents: a cross-sectional study. In: Gavião MB, Castelo PM LM. Factors Associated with Oral 2016. p. 137-48.

19. Barbosa TS, Gavião MB, Castelo PM, Leme MS. Factors associated with oral health-related quality of life in children and preadolescents: a cross-sectional study. Oral Health Prev Dent. 2016;14(2):137-48. 
20. Feitosa S, Colares V, Pinkham J. The psychosocial effects of severe caries in 4-year-old children in Recife, Pernambuco, Brazil. Cad Saúde Pública [Internet]. 2005 [acesso em 2017 Dez 20];21(5):1550-6. Disponível em: http://www.scielo.br/ scielo.php?script=sci_arttext\&pid=S0102-311X2005000500028\&lng=en\&nrm=iso\&tlng=en

21. McDonald RE. Odontopediatria. Roberval de Almeida Cruz, tradução. Rio de Janeiro: Guanabara Koogan; 2001.

22. Mattheus DJ. Efficacy of oral health promotion in primary care practice during early childhood: creating positive changes in parent's oral health beliefs and behaviors. Oral Health Dent Manag. 2014 Jun;13(2):316-9.

\section{Endereço do primeiro autor:}

Paula Reginne Vieira

Universidade Estadual Paulista - UNESP

Faculdade de Odontologia de Araraquara

Rua Humaitá, 1680

Bairro: Centro

CEP: 14801-903 - Araraquara - SP - Brasil

E-mail: paularvieira@foar.unesp.br

\section{Endereço para correspondência:}

Lourdes Aparecida Martins dos Santos-Pinto

Universidade Estadual Paulista - UNESP

Faculdade de Odontologia de Araraquara

Rua Humaitá, 1680

Bairro: Centro

CEP: 14801-903 - Araraquara - SP - Brasil

E-mail: 1spinto@foar.unesp.br 